\title{
Estructura y composición de ensamblajes de anfibios en tres tipos de hábitat en el Parque Nacional Natural Selva de Florencia, Cordillera Central de Colombia
}

\author{
Sebastián Duarte-Marín ${ }^{1,2, *}$, Cristian González-Acosta ${ }^{1,2}$, Fernando Vargas-Salinas ${ }^{1}$ \\ ${ }^{1}$ Grupo Evolución, Ecología y Conservación (EECO), Programa de Biología, Universidad del Quindío, Armenia, Colombia \\ ${ }^{2}$ Grupo de Herpetología de la Universidad del Quindío (GHUQ), Programa de Biología, \\ Universidad del Quindío, Armenia, Colombia
}

\begin{abstract}
Resumen
Los Andes colombianos, especialmente entre los 1000 y $3000 \mathrm{msnm}$, presentan la mayor diversidad de anfibios anuros para el país. Sin embargo, históricamente esta región ha tenido altos niveles de deforestación y cambios en el uso del suelo. Ante este escenario, el establecimiento de áreas protegidas es una importante estrategia de conservación de diversidad biológica en los Andes de Colombia. El Parque Nacional Natural (PNN) Selva de Florencia, está ubicado en la cordillera Central y es considerado el PNN con mayor diversidad de anfibios endémicos por área en el país; sin embargo, no se ha cuantificado la estructura y composición de los ensamblajes de anfibios ahí presentes de tal forma que, se establezca una línea base sólida para su monitoreo en espacio y tiempo. En este estudio cuantificamos la estructura y composición de los ensamblajes de anfibios en tres tipos de hábitat (bosque, pinera y pastizal), realizando muestreos diurnos y nocturnos entre junio y septiembre del 2017. Se registraron 338 individuos pertenecientes a 24 especies, 21 de ellas en bosque, 11 en pineras y 3 en pastizales. La diversidad de anfibios fue mayor en bosque que en pinera y potrero. La composición de especies en bosque tendió a ser diferente de aquella en pineras y potreros; sin embargo, en algunas pineras no sujetas a explotación comercial por más de 20 años, se observaron especies típicas de bosque. Nuestros resultados contribuyen con datos cuantitativos útiles para la monitorización de la diversidad de anfibios en los Andes centrales de Colombia, utilizando el PNN Selva de Florencia como modelo. (C) 2018. Acad. Colomb. Cienc. Ex. Fis. Nat.
\end{abstract}

Palabras clave: Anfibios; Ensamblajes; Endemismo; Selva de Florencia.

Structure and composition of amphibian assemblages in three types of habitat in the National Natural Park Selva de Florencia, Colombian Central Cordillera

\begin{abstract}
The Colombian Andes, especially between 1000 and 3000 masl, exhibit the greatest diversity of anuran amphibians for the whole country. However, historically this region has high levels of deforestation and changes in land use. Given this scenario, the establishment of protected areas is an important strategy for biological conservation in the Andes of Colombia. The National Natural Park (NNP) Selva de Florencia located in the Colombian Central Cordillera is considered the NNP with the greatest diversity of endemic amphibians per area in the country; however, the structure and composition of amphibian assemblages has not been quantified in such a way that a solid baseline data could be established for their monitoring both in space and time. In this study, the structure and composition of amphibian assemblages were quantified in three types of habitat (forest, pine crops and pasturelands), through diurnal and nocturnal samplings conducted between June and September 2017. A total of 338 individuals belonging to 24 species were registered, 21 of them in forest, 11 in pine crops, and 3 in pasturelands with the diversity of amphibians being greater in forest than in pine crops and pasturelands. The forest species composition differed from that in pine crops and pasturelands; nevertheless, in some pine crops with no commercial exploitation during the last 20 years, species common to the forest were recorded. Our results contribute with useful quantitative data for the monitoring of the amphibian diversity in the Colombian Central Cordillera using the NNP Selva de Florencia as a reference model. (C) 2018. Acad. Colomb. Cienc. Ex. Fis. Nat.
\end{abstract}

Key words: Amphibian; Assemblages; Endemism; Selva de Florencia.

\footnotetext{
*Correspondencia:

Sebastián Duarte-Marín, sdm950811@gmail.com, Recibido: 12 de marzo de 2018, Aceptado: 11 de mayo de 2018, Editor: Marta Ramírez Pinilla
} 


\section{Introducción}

Colombia está catalogada como uno de los países más ricos y diversos en fauna y flora (Myers, et al., 2000). Con respecto a los anfibios, en territorio colombiano se han registrado más de 800 especies $(>10 \%$ de las especies existentes a nivel global), lo que ubica a dicho país como el segundo más diverso en estos vertebrados después de Brasil (Acosta-Galvis, 2018; Frost, 2018). Esta diversidad no se encuentra uniformemente distribuida en Colombia; la región andina, especialmente aquella ubicada entre los 1000 y 3000 m, es la que exhibe la mayor diversidad de especies, muchas de ellas con rangos de distribución restringidos (Lynch, et al., 1997; Bernal \& Lynch, 2008; Hutter, et al., 2017). Lo anterior resalta la importancia de implementar estrategias de conservación de anfibios en la región andina de Colombia, región en la cual se concentra más del $66 \%$ de la población humana del país y que, por lo tanto, exhibe un alto nivel de deforestación y cambios en el uso del suelo (Rueda-Almonacid, 1999; Armenteras, et al., 2011).

Las áreas protegidas (parques o reservas naturales) son una estrategia útil para la conservación de ecosistemas naturales y de la biodiversidad en ellos existente (RangelChurio, 2006); dichas áreas pueden favorecer la persistencia de las poblaciones de anfibios al restringir actividades de origen antropogénico que afectarían negativamente la sobrevivencia y reproducción de los individuos (Lannoo, 2005). El Parque Nacional Natural (PNN) Selva de Florencia, ubicado en la cordillera Central de Colombia, es el PNN con mayor diversidad de especies de anfibios por área en todo el país; ya que con base en material colectado en este se han descrito más de 20 especies (Lynch \& Rueda-Almonacid, 1997, 1998a, 1998b, 1999; Rueda-Almonacid, et al., 2006; Ruiz-Carranza \& Lynch, 1997), muchas de ellas presentes solo en dicho lugar (Rueda-Almonacid, 2000; Ballesteros, et al., 2009). Sin embargo, los estudios con anfibios en el PNN Selva de Florencia no han hecho énfasis en la cuantificación de la estructura ni en la composición de los ensamblajes allí presentes, como tampoco han tratado su posible variación espacial de acuerdo con los tipos de hábitat (e.g. bosques, áreas de cultivo, potreros). Estudios que hacen esta cuantificación son frecuentes en la cordillera Occidental y Oriental (e.g. Cáceres-Andrade \& UrbinaCardona, 2009; Cortés, et al., 2008; Cubides \& UrbinaCardona, 2011; Cortés-Gómez, et al., 2013; GutiérrezLamus, et al., 2004; Méndez-Narváez \& Bolívar, 2016), pero son casi ausentes en la cordillera Central de Colombia (salvo por el informe de Hoyos-Hoyos, et al., 2012). Dado que los anfibios en los Andes colombianos exhiben rangos de distribución restringidos $\mathrm{y}$, por lo tanto, presentan un alto nivel de endemismo y recambio espacial de especies (Bernal \& Lynch, 2008; Meza-Joya \& Torres, 2016), es necesario estudiar directamente la respuesta de los ensamblajes de anfibios a perturbaciones antropogénicas en la cordillera Central, dado que las especies allí presentes no necesariamente exhiben los mismos niveles de susceptibilidad a cambios en su hábitat como las presentes en las otras dos cordilleras.

En este estudio se cuantificó la estructura y composición del ensamblaje de anfibios en tres tipos de hábitat (bosque, pinera y pastizales) presentes en el PNN Selva de Florencia. Para ello, utilizamos metodologías basadas en medidas de diversidad verdadera (sensu Jost, 2007) y análisis de cobertura de muestreo (Chao \& Jost, 2012), las cuales permiten comparar la diversidad de especies en espacio o tiempo sin incurrir en los sesgos presentes en metodologías tradicionalmente utilizadas para tal objetivo (Chao \& Jost, 2012). Por lo tanto, con el presente estudio esperamos contribuir con un análisis cuantitativo para la monitorización de la diversidad de anfibios en los Andes centrales de Colombia, utilizando como modelo el PNN Selva de Florencia.

\section{Materiales y métodos}

Area de estudio. El PNN Selva de Florencia está ubicado en el departamento de Caldas, específicamente en la vertiente oriental de la cordillera Central de Colombia. Este PNN tiene una extensión territorial de 10.019 hectáreas y está ubicado entre los 850 y $2400 \mathrm{~m}$, exhibe una temperatura media anual que oscila entre los 17 y $22^{\circ} \mathrm{C}$ y una precipitación mayor a los $8000 \mathrm{~mm} /$ año (Ballesteros, et al., 2009). Nuestra área específica de estudio está ubicada en áreas dentro de la jurisdicción del PNN Selva de Florencia y su zona de amortiguamiento en la vereda Las Colonias, Sector de Montebello (Ballesteros, et al., 2009) (Figura 1). Esta área se caracteriza por presentar ecosistemas de bosque húmedo altoandino y subandino con hábitats naturales bien conservados y otros de origen antropogénico, que acorde con su localización dentro o fuera de los límites del PNN Selva de Florencia, son utilizadas para aprovechamiento forestal y ganadero o han sido asignadas para permitir procesos de sucesión ecológica, respectivamente (Ballesteros et al., 2009).

Este estudio se realizó en tres tipos de hábitat (Figura 2). El hábitat bosque (BOS) se caracteriza por poseer numerosos árboles robustos $(\mathrm{DAP}>50 \mathrm{~cm})$ que forman un dosel de aproximadamente 30 metros de altura en los sitios menos perturbados, los árboles que exhiben poco epifitismo, el sotobosque es denso y con una capa delgada de hojarasca. El hábitat cultivos de pino (PIN) corresponde a sistemas de monocultivo para aprovechamiento forestal que forman un dosel de aproximadamente 20 metros de altura; predominan allí árboles de eucalipto y algunas plantas epifitas. El hábitat pastizales (PAS) corresponde a zonas de potrero utilizadas para cría de ganado, donde se encuentran charcas y cañadas temporales o permanentes con poca vegetación emergente y algunos márgenes de vegetación arbustiva y herbácea; los árboles robustos son dispersos y escasos.

Metodología. Entre junio y septiembre de 2017 se realizaron tres salidas de campo, cada una con duración de 12 días (Tabla 1). En cada una de ellas se muestrearon los tres tipos de hábitat seleccionados para este estudio, 


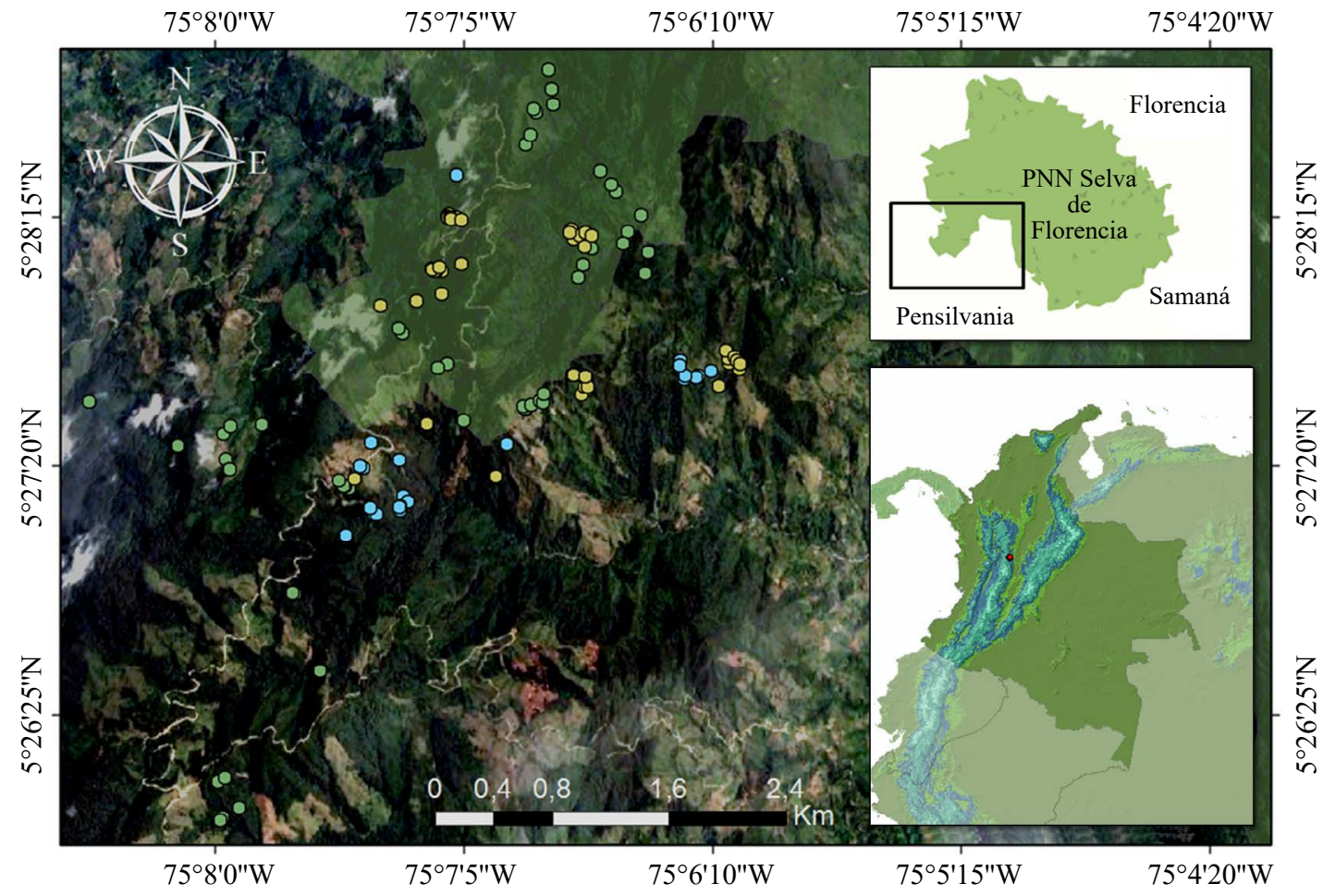

Figura 1. Imagen del PNN Selva de Florencia donde se señala el área de estudio y la ubicación de transectos en el hábitat de Bosque (puntos verdes), en Pineras (puntos azules) y Pastizal (puntos amarillos). Algunos puntos pueden estar sobrepuestos debido a la escala de la imagen.

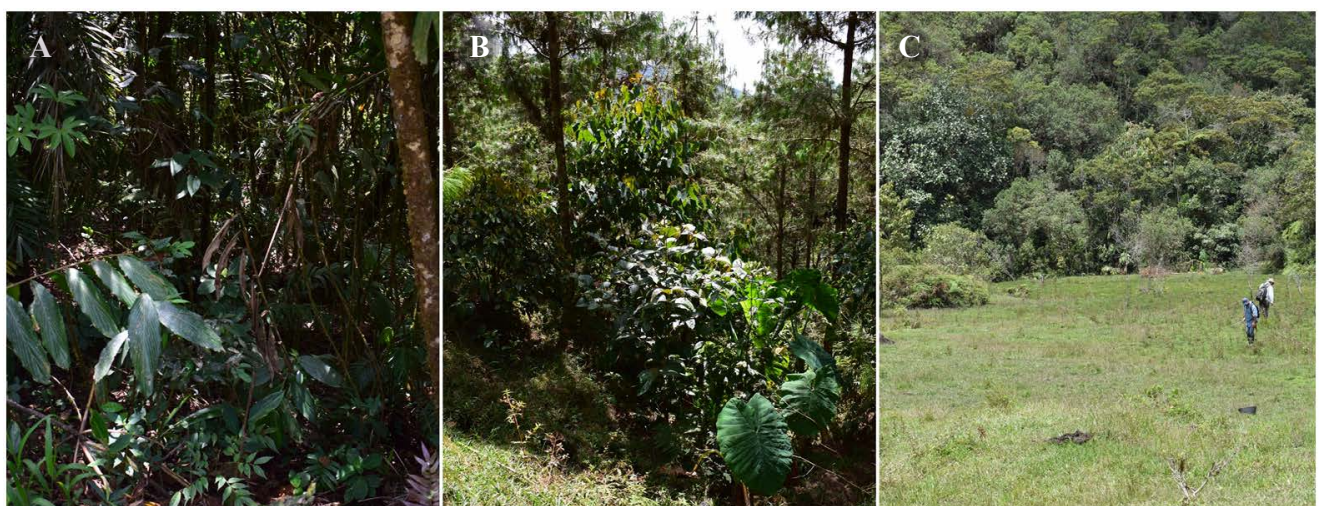

Figura 2. Tipos de hábitat muestreados en el PNN Selva de Florencia, Departamento de Caldas, Colombia. Bosque (A), Pinera (B) y Pastizal (C).

Tabla 1. Distribución y cantidad de transectos no permanentes establecidos en el Parque Nacional Natural Selva de Florencia. El número de transectos establecidos cerca de los cuerpos de agua se señalan con asterisco (*). Bosque (BOS), Pinera (PIN), Pastizal (PAS).

\begin{tabular}{lccc}
\hline Fecha & \multicolumn{3}{c}{ Hábitat } \\
\cline { 2 - 4 } & BOS & PIN & PAS \\
\hline 19-30 de junio & $19 * / 30$ & $3 * / 30$ & $5 * / 31$ \\
1-12 de agosto & $13 * / 25$ & $0 / 19$ & $7 * / 20$ \\
8-19 de septiembre & $10 * / 18$ & $2 * / 14$ & $1 * / 9$ \\
Total & $42 * / 73$ & $5 * / 63$ & $13 * / 60$ \\
\hline
\end{tabular}

utilizando transectos no permanentes de $100 \times 2$ metros. Los muestreos en cada transecto fueron llevados a cabo por tres personas tanto en el día ( $8 \mathrm{am}-11 \mathrm{am}$ ) como en la noche (7 pm - $1 \mathrm{am}$ ).

A cada individuo observado le fueron registrados los aspectos sugeridos por Heyer, et al., (1994) para caracterización de microhábitat, a saber, actividad en el momento de observación, tipo de sustrato (i.e. hoja, tronco, rama, suelo), altura de percha, y ubicación con respecto a cuerpos de agua (cerca, lejos). Además, se registró hora y fecha de encuentro, y se obtuvo la correspondiente georreferencia con un GPS Garmin Oregon 550. Para la identificación de 
especies se hizo un registro fotográfico de cada individuo (vista dorsal, ventral y lateral) con una cámara Nikon digital D5300. Algunos individuos que fueron colectados como vouchers fueron eutanizados con lidocaína al $2 \%$, se fijaron en formol al $10 \%$ y se preservaron en etanol al 70\% (Angulo, et al., 2006). A cada individuo colectado se le retiró un trozo de tejido (corte de extremidad posterior derecha), el cual se conservó en etanol al 96\% como muestra para futuros análisis genéticos acorde con los lineamientos sugeridos por Gonzalez \& Arenas-Castro (2017). Los individuos colectados y su respectiva muestra de tejido fueron depositados en la colección de Anfibios y Reptiles del Programa de Biología de la Universidad del Quindío, en Armenia, Colombia (Tabla 2). La clasificación taxonómica empleada en este estudio fue acorde con Frost (2018).

Análisis de datos. Para establecer y comparar la diversidad de especies entre BOS, PIN y PAS se realizó un análisis de cobertura de muestreo (Chao \& Jost, 2012) en la plataforma R v. 3.2 .5 (R Core Team, 2016) por medio del paquete iNEXT (Hsieh, et al., 2013). La cobertura de muestreo varía entre 0 y 1 , para indicar la probabilidad de que un próximo individuo capturado al azar perteneciera a una de las especies ya registradas en el muestreo. Además, extensiones de este análisis elaboradas por Colwell, et al., (2012) y Chao, et al., (2014) permitieron comparar la diversidad de especies (sensu Jost, 2007) entre hábitats, a la vez que se controló por sesgos existentes cada vez que las comparaciones se hacían por tamaño de muestra (principio de duplicidad sensu Chao \& Jost, 2012).

Los análisis de diversidad utilizan diferentes niveles de sensibilidad a la abudancia relativa de las especies $(\mathrm{q}=0$, $\mathrm{q}=1, \mathrm{q}=2$ ). Cuando $\mathrm{q}=0$, los cálculos no tienen en cuenta las diferencias en la abundancia de individuos y el resultado es equivalente al número de especies (i.e. riqueza). Cuando $\mathrm{q}=1$, se ponderan las especies acorde con su abundancia relativa y se obtiene un cálculo correspondiente al exponencial del índice de Shannon-Wienner. Cuando $q=2$, el resultado es influenciado principalmente por las especies más abundantes por lo que el cálculo corresponde al inverso del índice de Simpson (Jost, 2007; Jost \& GonzálezOreja, 2012).

Parar comparar el nivel de similitud en la composición de especies entre hábitats, se utilizó el índice de ChaoJaccard (Chao, et al., 2005, 2006) el cual, se calculó en Estimates v. 9.0 (Colwell, 2013) y se graficó en un dendograma utilizando PAST 3.0 (Hammer, et al., 2001). Este índice es una modificación del índice de similitud de Jaccard que es utilizado tradicionalmente en ecología de comunidades pero que permite corregir sesgos creados por tamaño de muestra y ausencia de registros de especies raras. Además, se calculó la diversidad beta por medio del índice propuesto por Jost (2007), el cual varía entre 1, cuando los ensamblajes a comparar son idénticos, y $\mathrm{N}$ (número de ensamblajes) cuando ellos son totalmente diferentes. Dado que la diversidad beta puede reflejar dos procesos diferentes y antitéticos (Anidamiento: especies de áreas con menor riqueza son subconjuntos de las especies de áreas con mayor riqueza $\left[\beta_{\mathrm{NES}}\right]$, o recambio espacial: remplazo de algunas especies por otras $\left[\beta_{\mathrm{SIM}}\right]$ ) (Baselga, 2010), calculamos los índices $\beta_{\mathrm{NES}}$ y $\beta_{\mathrm{SIM}}$ en el paquete estadístico betapart (Baselga \& Orme, 2012).

\section{Resultados}

Se registraron 24 especies de anfibios pertenecientes a dos órdenes y seis familias (Tabla 2; Figura 2S, https:// www.raccefyn.co/index.php/raccefyn/article/download SuppFile/631/2752). Cinco especies son endémicas para el PNN Selva de Florencia: Nymphargus spilotus, Andinobates daleswansoni, Pristimantis fetosus, P. tribulosus y P. veletis. Ocho especies se encuentran en alguna categoría de amenaza según la IUCN (2017); dos como Vulnerable (N. rosada, $A$. daleswansoni), cuatro En Peligro (Pristimantis actinolaimus, P. dorsopictus, P. fetosus, P. suetus), dos en Peligro Crítico (P. tribulosus, $P$. veletis) y una con Datos Deficientes ( $N$. spilotus). En estos registros y en los análisis cuantitativos de diversidad no fueron incluidos siete individuos de la rana marsupial Gastrotheca nicefori dado que fueron registrados con base en cantos y en áreas fuera de los transectos establecidos para nuestros muestreos.

Las características de microhábitat difirieron entre especies (Figura 3). Todas las especies, excepto P. w-nigrum y $A$. daleswansoni, fueron arbóreas, sin embargo, se registraron a diferentes rangos de altura y en diversos sustratos asociados al sotobosque. Los sustratos comúnmente utilizado por los individuos fueron hojas de herbáceas, arbustos o helechos. Otra característica del microhábitat en la cual se observó variación entre especies fue en la ubicación con respecto a cuerpos de agua. Diez especies fueron exclusivas de áreas aledañas a quebradas (e.g. Centrolene antioquiense, $N$. rosada, $P$. actinolaimus), siete especies fueron exclusivas de áreas alejadas de quebradas (e.g. A. daleswansoni, Bolitoglossa vallecula, $P$. uranobates), mientras que otras siete habitaron sitios tanto cerca como lejos de ellas (e.g. $P$. factiosus, P. suetus, P. tribulosus).

La cobertura de muestreo fue $\geq 0.95$ para cada uno de los tres tipos de hábitat (Figura 4). Respecto a la diversidad de orden $\mathrm{q}=0$, esta fue estadísticamente mayor en BOS, intermedia en PIN y más baja en PAS. Sin embargo, para los estimados de diversidad de orden $\mathrm{q}=1$ y $\mathrm{q}=2$, la diversidad entre PIN y PAS no difirió; BOS siguió siendo significativamente más diversa que PIN y PAS. La estructura del ensamblaje de anfibios en BOS fue menos heterogénea con respecto a la abundancia relativa de las especies que la estructura en PIN y PAS, puesto que en cada una predominó una especie muy abundante (P. factiosus y P. erythropleura, respectivamente) (Figura 5).

Respecto al recambio de especies entre hábitats, el índice de diversidad beta $(=2.20)$ y los resultados obtenidos con el análisis de agrupamiento (Figura 1S, https://www.raccefyn. co/index.php/raccefyn/article/downloadSuppFile/631/2751), 
Tabla 2. Riqueza, hábitat, estado de conservación y número de colección de los anfibios registrados en el PNN Selva de Florencia, Pensilvania, Departamento de Caldas, Colombia. Arb $=$ arbórea, Terr $=$ terrestre, Diur $=$ diurna, $=$ Noct $=$ nocturna, $\mathrm{BOS}=\mathrm{bosque}, \mathrm{PIN}=$ Pinera, PAS= pastizal. Las especies endémicas para el PNN Selva de Florencia se señalan con asterisco (*). Nivel de amenaza nacional (NAL) acorde a la Resolución No. 1912 del 2017 y el internacional (INT) acorde a la IUCN (http://www.iucnredlist.org). Imagen de las especies observadas en este estudio son presentadas en la figura 2S. ARUQ se refiere al código del espécimen (vouchers) en la colección de anfibios y reptiles de la Universidad del Quindío, Colombia.

\begin{tabular}{|c|c|c|c|c|c|c|c|}
\hline \multirow[t]{2}{*}{ TAXA } & \multirow[t]{2}{*}{ Actividad } & \multicolumn{3}{|c|}{ Hábitat } & \multicolumn{2}{|c|}{$\begin{array}{c}\text { Nivel de } \\
\text { amenaza }^{1}\end{array}$} & \multirow{2}{*}{$\begin{array}{l}\text { Voucher** } \\
\text { ARUQ }\end{array}$} \\
\hline & & BOS & PIN & PAS & NAL & INT & \\
\hline $\begin{array}{l}\text { Orden Caudata } \\
\text { Plethodontidae } \\
\text { Bolitoglossa vallecula Brame \& Wake, } 1963\end{array}$ & Arb-Noct & & 2 & & & $\mathrm{LC}$ & 785 \\
\hline $\begin{array}{l}\text { Orden Anura } \\
\text { Bufonidae } \\
\text { Rhinella aff. macrorhina }\end{array}$ & Terr-Noct & 1 & & & & & 762 \\
\hline $\begin{array}{l}\text { Centrolenidae } \\
\text { Centrolene antioquiense Noble, } 1920\end{array}$ & Arb-Noct & 8 & 1 & & & NT & $789,807,808$ \\
\hline Nymphargus grandisonae Cochran \& Goin, 1970 & Arb-Noct & 2 & & & & $\mathrm{LC}$ & \\
\hline Nymphargus rosada Ruiz-Carranza \& Lynch, 1997 & Arb-Noct & 6 & & & & VU & 805,806 \\
\hline Nymphargus spilotus Ruiz-Carranza \& Lynch, 1997* & Arb-Noct & 4 & & & & $\mathrm{DD}$ & $794,795,796,797$ \\
\hline $\begin{array}{l}\text { Dendrobatidae } \\
\text { Andinobates daleswansoni Rueda-Almonacid, } \\
\text { et al., 2006* }\end{array}$ & Terr-Diur & 13 & 2 & & & VU & $767,768,775$ \\
\hline $\begin{array}{l}\text { Hylidae } \\
\text { Hyloscirtus larinopygion Duellman, } 1973\end{array}$ & Arb-Noct & 4 & & & & NT & 769,773 \\
\hline $\begin{array}{l}\text { Strabomantidae } \\
\text { Pristimantis actinolaimus Lynch \& Rueda- } \\
\text { Almonacid, } 1998\end{array}$ & Arb-Noct & 4 & & & VU & EN & 771 \\
\hline Pristimantis boulengeri Lynch, 1981 & Arb-Noct & 1 & & & & & $800,801,802,803,804$ \\
\hline Pristimantis dorsopictus Rivero \& Serna, 1988 & Arb-Noct & 19 & 1 & & & EN & $759,760,811$ \\
\hline Pristimantis erythropleura Boulenger, 1986 & Arb/Terr-Noct & 2 & 3 & 22 & & $\mathrm{LC}$ & 778 \\
\hline Pristimantis factiosus Lynch \& Rueda-Almonacid, 1998 & Arb-Noct & 38 & 98 & & & $\mathrm{LC}$ & 772,784 \\
\hline Pristimantis fetosus Lynch \& Rueda-Almonacid, 1998 * & Arb-Noct & 6 & & & VU & EN & $788,798,799$ \\
\hline Pristimantis suetus Lynch \& Rueda-Almonacid, 1998 & Arb-Noct & 10 & 1 & & VU & EN & 763,813 \\
\hline Pristimantis taeniatus Boulenger, 1912 & Arb-Noct & 6 & 22 & 9 & & $\mathrm{LC}$ & $\begin{array}{l}780,781,790 \\
809,810\end{array}$ \\
\hline $\begin{array}{l}\text { Pristimantis tribulosus Lynch \& Rueda-Almonacid, } \\
1997 \text { * }\end{array}$ & Arb-Noct & 5 & 4 & & $\mathrm{EN}$ & $\mathrm{CR}$ & $761,776,822$ \\
\hline $\begin{array}{l}\text { Pristimantis cf. permixtus Lynch, Ruiz-Carranza \& } \\
\text { Ardila-Robayo, } 1994\end{array}$ & Terr-Noct & 1 & & & & $\mathrm{LC}$ & 812 \\
\hline Pristimantis uranobates Lynch, 1991 & Arb-Noct & 8 & & & & $\mathrm{LC}$ & 777 \\
\hline Pristimantis veletis Ruiz-Carranza \& Lynch, 1997 * & Arb-Noct & & 4 & & $\mathrm{EN}$ & $\mathrm{CR}$ & 779,791 \\
\hline Pristimantis w-nigrum Boettger 1892 & Terr-Noct & & & 7 & & $\mathrm{LC}$ & 786,787 \\
\hline Pristimantis sp. 1 & Arb-Noct & 1 & & & & & 764 \\
\hline Pristimantis sp. 2 & Arb-Noct & 1 & & & & & 765 \\
\hline Pristimantis sp. 3 & Arb-Noct & 3 & 3 & & & & $\begin{array}{l}814,815,816 \\
817,818,819\end{array}$ \\
\hline Número de individuos por hábitat & & 159 & 141 & 38 & & & \\
\hline
\end{tabular}

$1 \mathrm{CR}=$ Peligro crítico, $\mathrm{VU}=$ Vulnerable, $\mathrm{LC}=$ preocupación menor, $\mathrm{NT}=$ casi amenazado, $\mathrm{DD}=$ Datos deficientes. 

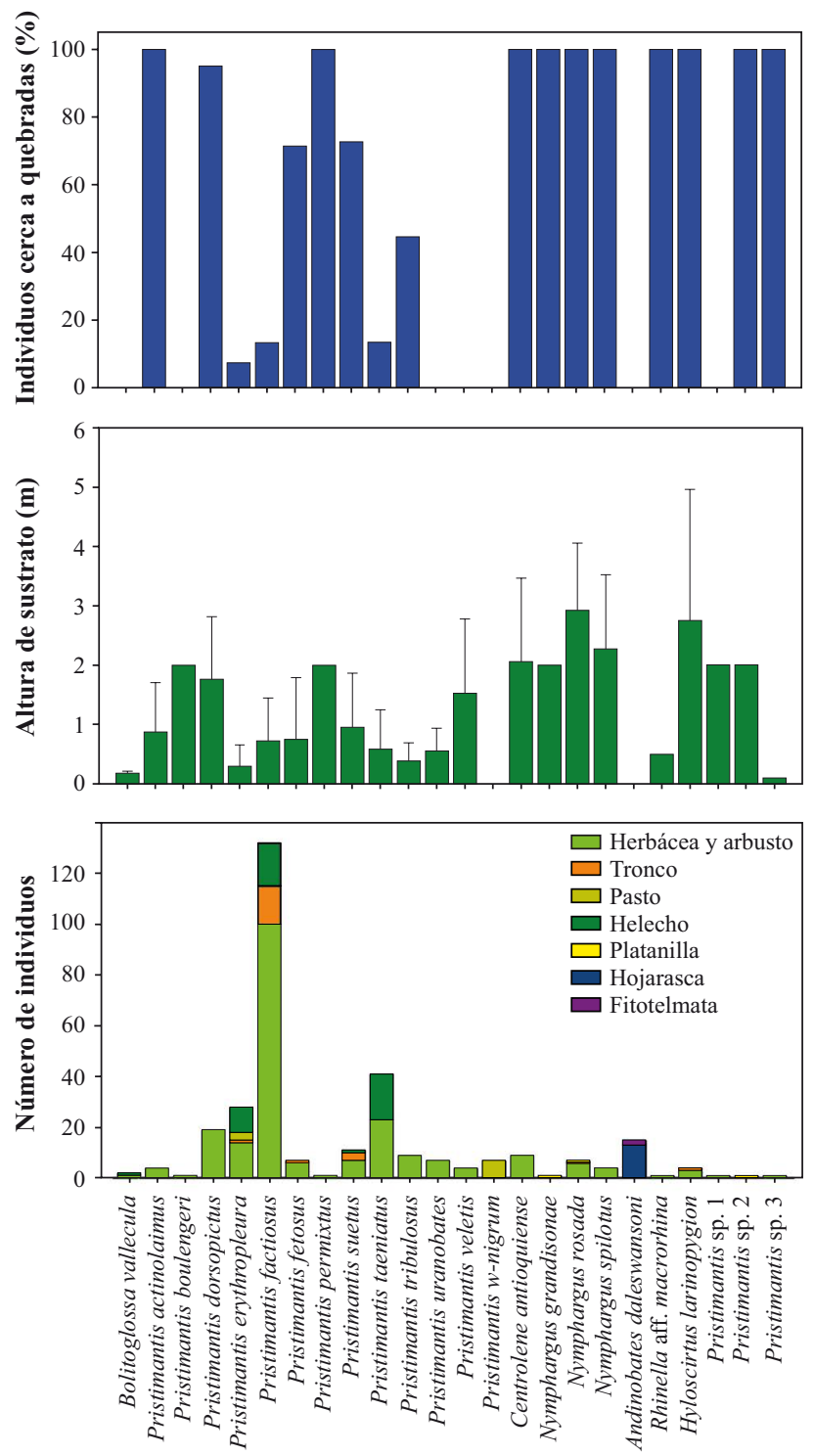

Figura 3. Características de microhábitat de los anfibios registrados en el PNN Selva de Florencia. Gráfica superior: ubicación de individuos por especie respecto a cuerpos de agua (quebradas); gráfica intermedia: altura promedio del sustrato en que se observaron los individuos (rangos indican desviación estándar); gráfica inferior: tipo de sustrato en que se encontraron los individuos.

mostraron dos entidades o grupos diferenciados, uno conformado por el ensamblaje de anfibios en PAS y el otro representado por el ensamblaje en PIN y BOS. Un resultado de $\beta_{\mathrm{NES}}=0.4$ y de $\beta_{\mathrm{SIM}}=0.27$, sugiere que las diferencias en composición de especies entre hábitats eran debidas más a un proceso de anidamiento que a un proceso de reemplazo espacial de especies.

\section{Discusión}

Reportamos 24 especies de anfibios, 20 de ellas registradas previamente para el área de estudio (Rueda-Almonacid, 2000); entre las cuatro especies adicionales están tres de la familia Strabomantidae y una de Centrolenidae. Las tres especies de Strabomantidae no han podido ser asociadas con ningún taxón en particular ya que ellas exhiben variaciones morfológicas importantes que podrían implicar nuevos reportes para la fauna de anfibios del PNN Selva de Florencia o incluso, especies aun no descritas. La especie de Centrolenidae corresponde a $N$. spilotus, rana de cristal endémica del PNN Selva de Florencia, la que solo se había registrado en su localidad tipo ("El Estadero", corregimiento de Florencia, municipio de Samaná) localizado a $10.2 \mathrm{Km}$ lineales de nuestra área de estudio (Ruíz-Carranza \& Lynch, 1997). Otras once especies conocidas en el sector de Montebello no fueron registradas en nuestro estudio (Centrolene geckoideum, C. robledoi, Hemiphractus johnsoni Hyloscirtus aff. simmonsi, Atelopus sp. nov., Pristimantis cf. cabrerai, $P$. lichenoides, $P$. maculosus, $P$. parectatus, $P$ torrenticola, Strabomantis necopinus). Tres de estas especies (Atelopus sp. nov., $P$. lichenoides y $P$. torrenticola) no han sido observadas desde 1994 (Rueda-Almonacid, 2000), lo que genera especial preocupación ya que son endémicas del PNN Selva de Florencia. La ausencia del registro de las otras ocho especies puede deberse a varias razones no excluyentes entre sí, por ejemplo, que sus poblaciones aparentemente exhiben baja densidad de individuos y han sido registradas en localidades específicas no abarcadas en este estudio (Rueda-Almonacid, 2000).

Aunque la cobertura de muestreo fue alta para cada hábitat $(\geq 0.95)$, el análisis sugiere que la diversidad de especies en el área de estudio puede aún incrementarse. Además, basados en la relación área - riqueza de especies (Rosenzweig, 1995), es muy probable que estudios futuros en zonas poco muestreadas y de difícil acceso, incrementen la diversidad de anfibios en el PNN Selva de Florencia. Dicha diversidad pudiera incluir especies no descritas formalmente para la ciencia, o especies registradas en sitios cercanos, por ejemplo, Atopophrynus syntomopus (Lynch \& RuizCarranza, 1982), Colostethus thorntoni (Cochran \& Goin, 1970), Hyloscirtus antioquia (Rivera-Correa \& Faivovich, 2013) o Pristimantis scoloblepharus (Lynch, 1991).

La mayor diversidad de anfibios en BOS que en PIN y PAS, se atribuye a una mayor complejidad vegetal y cobertura de dosel en BOS. Lo anterior se traduciría en mayor disponibilidad de microhábitats húmedos a nivel del sotobosque, lo cual, es particularmente importante para especies de anfibios con ovoposición fuera del agua, como lo son aquellas presentes en el área de estudio. De hecho, las especies en PAS estuvieron principalmente asociadas a vegetación herbácea y arbustiva, que incluso en zonas sin cobertura de dosel alta, podrían ayudar a mantener una capa de hojarasca y microhábitats húmedos a nivel del suelo. Un patrón similar de incremento en la diversidad de especies de anfibios hacia coberturas boscosas y con mayor complejidad vegetal se ha reportado en otros estudios realizados tanto en la cordillera Occidental (Cortés-Gómez, et al., 2013; Cubides \& Urbina-Cardona, 2011; García-R, et al., 2005; 


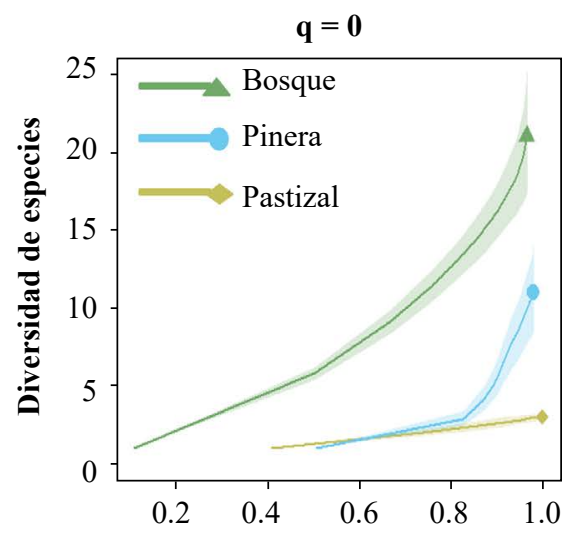

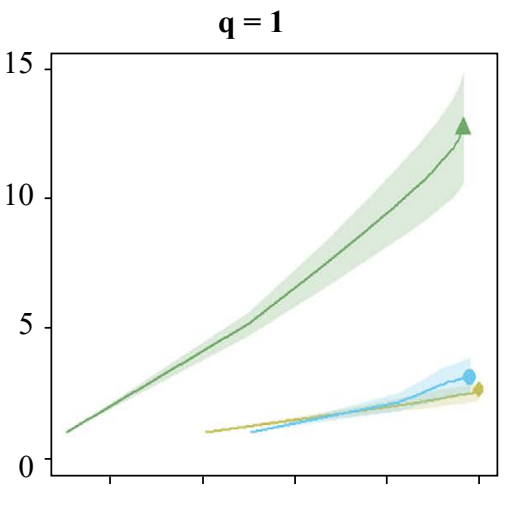

Cobertura de muestreo

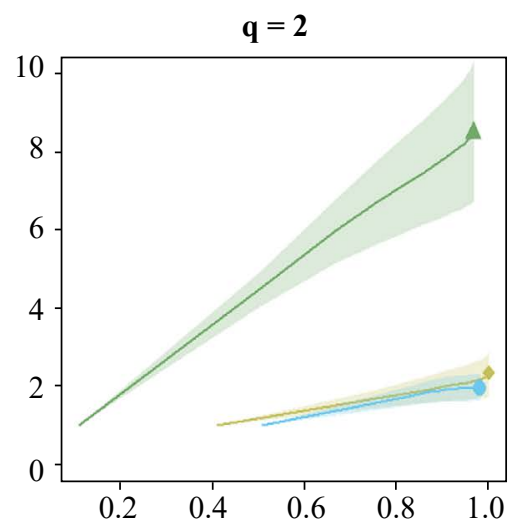

Figura 4. Relación entre cobertura de muestreo y diversidad de especies de anfibios en Bosque, Pinera y Pastizal. Los valores de " $q$ " indican el nivel de sensibilidad de los cálculos de diversidad a la abundancia relativa de las especies (ver texto en Metodología para detalles). Las áreas sombreadas señalan los intervalos de confianza del $95 \%$ correspondiente a cada tipo de hábitat.

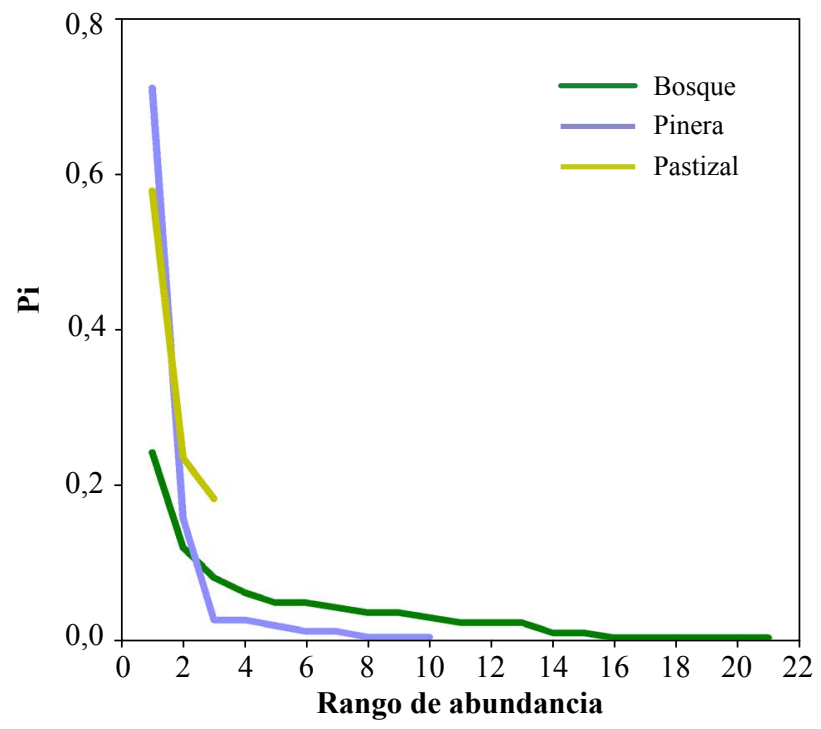

Figura 5. Grafica de rango de abundancia que señala diferencias en la estructura de los ensamblajes de anfibios en los tres hábitats incluidos en este estudio. $\mathrm{Pi}=$ Número de individuos especie $i$ / Numero de individuos de todas las especies.

Méndez-Narváez \& Bolívar, 2016) como en la cordillera Oriental de Colombia (Cáceres-Andrade \& UrbinaCardona, 2009; Gutiérrez-Lamus, et al., 2004; Cortés et al., 2008).

Los análisis de composición de especies estuvieron acordes con el patrón que reportamos respecto a la estructura de ensamblajes. Por un lado, las especies de BOS requieren coberturas vegetales densas, algunas de ellas claramente asociadas con cuerpos de agua lóticos; por ejemplo, N. spilotus, $N$. grandisonae, N. rosada, C. antioquensis, Hyloscirtus larinopygion y Pristimantis dorsopictus (Rivera-Correa \& Faivovich, 2013; Rada, et al., 2017). Por otro lado, las especies registradas en PAS y PIN (i.e. $P$. erythropleura, $P$. taeniatus y $P$. w-nigrum) pueden soportar perturbación en su hábitat, aunque en estos hábitats deben estar presentes mircrohábitats lo suficientemente húmedos para su reproducción, y para ofrecer refugio e ítems alimenticios (Montes, et al., 2004). Un aspecto a resaltar es el registro en PIN de especies habitualmente consideradas como anfibios de zonas de bosque conservado: A. daleswansoni, $B$. vallecula, $P$. tribulosus y $P$. veletis. Además, algunas de estas especies son categorizadas como amenazadas (IUCN, 2017). Lo anterior es acorde con nuestros resultados de $\beta_{\mathrm{NES}}$ y sugiere que PIN provee los recursos suficientes para la subsistencia de un subconjunto de las especies típicas de bosque. Por el contrario, en hábitats cuya cobertura de dosel y heterogeneidad vegetal es altamente reducida (e.g. PAS), empieza a darse un reemplazo de especies de áreas boscosas por especies típicas de áreas abiertas; este proceso es reflejado cuantitativamente en nuestro resultado de $\beta_{\text {SIM }}$.

Actualmente, se resalta el valor que, en conservación de anfibios, podrían estar jugando bosques secundarios o hábitats perturbados donde se ha permitido que avancen procesos de sucesión ecológica (Herrera-Montes \& Brokaw, 2010; Hernández-Ordoñez, et al., 2015; Hilje \& Aide, 2012; Russildi, et al., 2016; Zermeño-Hernández, et al., 2016). Sin embargo, algunos estudios no han arrojado resultados que respalden esta hipótesis (Gardner, et al., 2007a, 2007b; Vallan, 2002). La disposición espacial entre hábitats en sucesión y bosques bien conservados que sirvan de fuente de especies ha sido una explicación factible para argumentar por qué la evidencia a este respecto ha sido contradictoria (Pough, et al., 2016). En el caso particular del PNN Selva de Florencia, algunas pineras (PIN) han estado sin aprovechamiento forestal durante casi 20 años (Ballesteros, et al., 2009) permitiendo un incremento en densidad de sotobosque y complejidad de la estructura vegetal (observación personal) que iría acompañada de la recuperación paulatina de los ensamblajes de anfibios, y quizás de otros grupos animales (Johnston \& Odun, 1956; Rios-López, 2007; Cortés-Gómez, et al., 2013). Las áreas 
de PIN donde se registraron las especies típicas de bosque están adyacentes o rodeadas por bosque, mientras que en áreas de PIN bordeadas por pastizales solo se observaron especies típicas de áreas abiertas y perturbadas (e.g. $P$. w-nigrum, P. erythropleura, P. taeniatus).

Un aspecto importante a resaltar es que en nuestros muestreos, tres especies endémicas al PNN Selva de Florencia (P. actinolaimus, $P$. fetosus y $P$. veletis) fueron registradas exclusivamente en transectos localizados fuera de los límites legales del PNN. Aunque esto no significa que estas especies no sigan estando presentes dentro del PNN Selva de Florencia (Lynch \& Rueda-Almonacid, 1997, 1998a; Rueda-Almonacid, 2000) si resalta la importancia de implementar planes de manejo en su área de amortiguamiento con el objetivo de fortalecer las estrategias de conservación de los anfibios en la región. Más aún, en dichas áreas de amortiguamiento también se registraron otras especies de anfibios endémicas para el PNN Selva de Florencia: P. tribulosus y A. daleswansoni.

Actualmente, Colombia experimenta una significativa reducción en los tamaños poblacionales de anfibios (RuedaAlmonacid, et al., 2005; Flechas, et al., 2017), lo que se atribuye principalmente a deforestación, cambio climático y enfermedades emergentes (Becker, et al., 2007; Ruiz \& Rueda-Almonacid, 2008; Urbina-Cardona, 2016). La presencia del PNN Selva de Florencia ayuda a controlar lo que tradicionalmente se ha considerado la causa más importante de pérdida de biodiversidad (i.e. deforestación); sin embargo, los ensamblajes de anfibios ahí presentes no están exentos de los efectos atribuidos a cambio climático y a la presencia del hongo Batrachochytrium dendrobatidis. Por lo tanto, estudios futuros deberían abarcar la monitorización de poblaciones y ensamblajes de anfibios para evaluar el potencial efecto de estos dos factores. Nuestro estudio ofrece información cuantitativa que servirá de base para dichos monitoreos y los efectos de regeneración natural que actualmente se permiten en el PNN Selva de Florencia.

\section{Conclusiones}

La estructura y composición de los ensamblajes de anfibios en bosque (BOS) difiere de aquella en pineras (PIN) y pastizales (PAS). La presencia de especies tradicionalmente asignadas a hábitats boscosos y catalogados con algún grado de amenaza en el hábitat de PIN, sugiere que si los procesos de sucesión ecológica siguen avanzando en este hábitat, ahí se podrían encontrar ensamblajes cada vez más diversos y similares a los presentes en BOS. Por el contrario, en ambientes muy perturbados y cuya heterogeneidad vegetal es altamente reducida, empieza a darse un reemplazo de especies de áreas boscosas por aquellas típicas de áreas abiertas.

\section{Agradecimientos}

Gracias a Parques Nacionales Naturales de Colombia y a Wildlife Conservation Society WCS (bajo el Fondo de Investigación Mono Hernández) por el apoyo financiero y logístico para la realización de este estudio. Igualmente, gracias a Hugo Ballesteros, Catherine Rodríguez Hurtado, Amilvia Acosta Castañeda, Andrés Felipe Toro Cardona, Johan A. Giraldo Romero, Ricardo A. García Arango, Milton Pineda Duque, José Alzate Henao y Paula A. NavarroSalcedo, por su invaluable ayuda en el trabajo de campo. Finalmente, agradecemos a Gustavo González-Duran por su ayuda en la determinación taxonómica de las especies, a Martha Patricia Ramírez-Pinilla y a dos evaluadores anónimos por sus comentarios y sugerencias para mejorar este manuscrito.

\section{Contribución de los autores}

Sebastián Duarte-Marín concibió el estudio. Sebastián Duarte-Marín y Fernando Vargas-Salinas establecieron el diseño de muestreo y los análisis de datos. Sebastián DuarteMarín y Cristian González-Acosta realizaron la toma de datos en campo. Todos los autores participaron en la escritura del manuscrito.

\section{Información suplementaria}

Figura 1S. Nivel de similitud entre tres tipos de hábitat en el PNN Selva de Florencia con base en la composición de anfibios presentes en ellos. Medida de distancia basada en el índice de Chao-Jaccard. Vea la figura 1S en: https:/www.raccefyn.co/index.php/raccefyn/ article/downloadSuppFile/631/2751

Figura 2S. Imagen de los anfibios registrados en el PNN Selva de Florencia, Departamento de Caldas. Vea la figura 2S en: https://www. raccefyn.co/index.php/raccefyn/article/downloadSuppFile/631/2752

\section{Conflicto de intereses}

Los autores declaran que no tienen conflictos de intereses con respecto al contenido del artículo.

\section{Referencias}

Acosta-Galvis, A. R. (2018). Lista de los Anfibios de Colombia. Batrachia, Villa de Leyva, Boyacá, Colombia. Disponible en: http://www.batrachia.com, accedida entre enero y marzo de 2018.

Angulo, A., Rueda-Almonacid, J. V. Rodríguez-Mahecha, J. V., La Marca E. (2006). Técnicas de inventario y monitoreo para los anfibios de la región tropical andina. Conservación Internacional. Serie Manuales de Campo No 2. Bogotá, Colombia: Panamericana Formas e Impresos S.A. 300 pp.

Armenteras, D., Rodríguez, N., Retana, J., Morales, M. (2011). Understanding deforestation in montane and lowland forests of the Colombian Andes. Regional Environmental Change, 11: 693-705.

Ballesteros, H., Arroyave, J. F., Walker, R., Echeverry, L., Acosta, H., Betancourt, A. F., Diaz-Mesa, J., Lopez, M. P., Moreno-Ortiz, E., Villegas, H., Hincapié, W., Lancheros, R., Patiño, M. (2009). Plan de manejo 2008 - 2012 Parque Nacional Natural Selva de Florencia. Corregimiento de Florencia - Samaná (Caldas): Parques Nacionales Naturales de Colombia, 141 pp. 
Baselga, A. (2010). Partitioning the turnover and nestedness components of beta diversity. Global Ecology and Biogeography. 19 (1): 134-143.

Baselga, A., Orme, C. D. L. (2012). betapart: an R package for the study of beta diversity. Methods in Ecology and Evolution. 3 (5): 808-812.

Becker, C., Fonseca, C., Baptista, C., Baptista, R., Prado, P. (2007). Habitat split and the global decline of amphibians. Science. 318: 1775-1777.

Bernal, M. H., Lynch, J. D. (2008). Review and analysis of altitudinal distribution of the Andean anurans in Colombia. Zootaxa. 1826 (1): 1-25.

Cáceres-Andrade, S. P., Urbina-Cardona, J. N. (2009). Ensamblajes de anuros de sistemas productivos y bosques en el piedemonte llanero, Departamento del Meta, Colombia. Caldasia. 31 (1): 175-194.

Chao, A., Jost, L. (2012). Coverage-based rarefaction and extrapolation: standardizing samples by completeness rather than size. Ecology. 93: 2533-2547.

Chao, A., Gotelli, N., Hsieh, T., Sander, E., Ma, K., Colwell, R., Ellison, A. (2014). Rarefaction and extrapolation with Hill numbers: a framework for sampling and estimation in species diversity studies. Ecological Monographs. 84: 45-67.

Cochran, D. M., Goin, C. J. (1970). Frogs of Colombia. Bulletin of the United States National Museum. 288: 1-655.

Colwell, R., Chao, A., Gotelli, N., Lin, S., Mao, C., Chazdon, R., Longino, J. (2012). Models and estimators linking individual-based and sample-based rarefaction, extrapolation and comparison of assemblages. Journal of Plant Ecology. 5: 3-21.

Colwell, R. (2013). EstimateS: Statistical estimation of species richness and shared species from samples. Version 9.1. User's Guide and application. Disponible en: http://viceroy.eeb. uconn.edu/estimates/, accedida entre enero y junio de 2018.

Cortés, A. M., Ramírez-Pinilla, M. P., Suárez, H. A., \& Tovar, E. (2008). Edge effects on richness, abundance and diversity of frogs in Andean cloud forest fragments. South American Journal of Herpetology, 3 (3): 213-222.

Cortés-Gómez, A. M., Castro-Herrera, F., Urbina-Cardona, J. N. (2013). Small changes in vegetation structure create great changes in amphibian ensembles in the Colombian Pacific rainforest. Tropical Conservation Science. 6 (6): 749-769.

Cubides, P. I., Urbina-Cardona, J. (2011). Anthropogenic disturbance and edge effects on anuran assemblages inhabiting cloud forest fragments in Colombia. Natureza \& Conservacao. 9: 39-46.

Flechas, S. V., Paz, A., Crawford, A. J., Sarmiento, C., Acevedo, A. A., Arboleda, A., Bolívar-García, W., Echeverry-Sandoval, C. L., Franco, R., Mojica, C., Muñoz, A., Palacios-Rodríguez, P., Posso-Terranova, A. M., Quintero-Marín, P., Rueda-Solano, L. A., CastroHerrera, F., Amézquita, A. (2017). Current and predicted distribution of the pathogenic fungus Batrachochytrium dendrobatidis in Colombia, a hotspot of amphibian biodiversity. Biotropica. 49 (5): 685-694.

Frost, D. R. (2018). Amphibian Species of the World: an Online Reference. Version 6.0, American Museum of Natural History, New York, USA. Disponible en: http://research. amnh.org/herpetology/amphibia/index.html., accedida entre enero y junio de 2018.
García-R, J. C., Castro-H, F., Cárdenas-H, H. (2005). Relación entre la distribución de anuros y variables del hábitat en el sector La Romelia del Parque Nacional Natural Munchique (Cauca, Colombia). Caldasia. 27 (2): 299-310.

Gardner, T. A., Barlow, J., Peres, C. A. (2007a). Paradox, presumption and pitfalls in conservation biology: the importance of habitat change for amphibians and reptiles. Biological Conservation. 138 (1-2): 166-179.

Gardner, T. A., Ribieiro-Júnior, M. A., Barlow, J. O. S., Ávila $\square$ Pires, T. C. S., Hoogmoed, M. S., Peres, C. A. (2007b). The value of primary, secondary, and plantation forests for a neotropical herpetofauna. Conservation biology. 21 (3): 775-787.

Gonzalez M. A., Arenas-Castro, H. (2017). Recolección de tejidos biológicos para análisis genéticos. Bogotá, Colombia.: Instituto de Investigación de Recursos Biológicos Alexander von Humboldt, 33 pp.

Gutiérrez-Lamus, D. L., Serrano, V. H., Ramírez-Pinilla, M. P. (2004). Composición y abundancia de anuros en dos tipos de bosque (natural y cultivado) en la Cordillera Oriental colombiana. Caldasia. 26 (1): 245-264.

Hammer, Ø., Harper, D. A. T., Ryan, P. D. (2001). PAST: Paleontological Statistics software package for education and data analysis. Paleontología Electronica. 4: 1-9.

Hernández-Ordóñez, O., Urbina-Cardona, N., MartínezRamos, M. (2015). Recovery of amphibian and reptile assemblages during old-field succession of tropical rain forests. Biotropica. 47 (3): 377-388.

Herrera-Montes, A., Brokaw, N. (2010). Conservation value of tropical secondary forest: a herpetofaunal perspective. Biological Conservation. 143 (6): 1414-1422.

Heyer, W. R., Donelly, M. A., Mc Diarmid, R. W., Hayek, L. C., Foster, M. S. (1994). Measuring and monitoring biological diversity: standard methods for amphibians. Washington, USA: Smithsonian Institution Press, 364 pp.

Hilje, B., Aide, T. M. (2012). Recovery of amphibian species richness and composition in a chronosequence of secondary forests, northeastern Costa Rica. Biological Conservation. 146 (1): 170-176.

Hoyos-Hoyos, J. M., Isaacs-Cubides, P., Devia, N., GalindoUribe, D. M., Acosta-Galvis, A. R. (2012). An approach to the ecology of the herpetofauna in agroecosystems of the Colombian coffee zone. South American Journal of Herpetology. 7 (1): 25-34.

Hsieh, T., K. Ma., Chao, A. (2013). iNEXT online: interpolation and extrapolation. Version 1.3.0. Disponible en: http:// glimmer.rstudio.com/tchsieh/inext/, accedida entre enero y junio de 2018.

Hutter, C. R., Lambert, S. M., Wiens, J. J. (2017). Rapid diversification and time explain amphibian richness at different scales in the Tropical Andes, Earth's most biodiverse hotspot. The American Naturalist. 190 (6): 828-843.

IUCN. (2017). The IUCN Red List of Threatened Species. Versión 2017-3. Disponible en: http://www.iucnredlist.org, accedida entre enero y junio de 2018.

Johnston, W. D., Odum, E. P. (1956): Breeding bird populations in relation to plant succession on the piedmont of Georgia. Ecology. 37: 50-62.

Jost, L. (2007). Partitioning diversity into independent alpha and beta components. Ecology. 88: 2427-2439. 
Jost, L., González-Oreja, J. A. (2012). Midiendo la diversidad biológica: más allá del índice de Shannon. Acta Zoológica Lilloana. 56: 3-14.

Lannoo, M. J. (2005). Amphibian declines: the conservation status of United States species. California, USA: University of California Press, 1115 pp.

Lynch, J. D. (1991). New diminutive Eleutherodactylus from the cordillera Central of Colombia (Amphibia: Leptodactylidae). Journal of Herpetology. 25: 344-352.

Lynch, J. D. Rueda-Almonacid, J. V. (1997). Tres nuevas especies de Eleutherodactylus: (Leptodactylidae) de los bosques nublados del este del Departamento de Caldas, Colombia. Revista de la Academia Colombiana de Ciencias Exactas, Físicas y Naturales. 21 (79): 131-142.

Lynch, J. D., Rueda-Almonacid, J. V. (1998a). Especies adicionales de ranas (género Eleutherodactylus) de los bosques nublados del Oriente del departamento de Caldas, Colombia. Revista de la Academia Colombiana de Ciencias Exactas, Físicas y Naturales. 22 (83): 287-298.

Lynch, J. D., Rueda-Almonacid, J. V. (1998b). Nuevas ranas del género Eleutherodactylus de la vertiente oriental del norte de la Cordillera Central, Colombia. Revista de la Academia Colombiana de Ciencias Exactas, Físicas y Naturales. 22 (85): 561-570.

Lynch, J. D., Rueda-Almonacid, J. V. (1999). Nuevas especies de ranas de altitudes baja y media en un perfil en el flanco oriental de la cordillera Central del Departamento de Caldas. Revista de la Academia Colombiana de Ciencias Exactas, Físicas y Naturales. 23 (87): 307-314.

Lynch, J. D., Ruiz-Carranza, P. M. (1982). A new genus and species of poison dart frog (Amphibia: Dendrobatidae) from the Andes of northern Colombia. Proceedings of the Biological Society of Washington. 95: 557-562.

Lynch, J. D., Ruiz-Carranza, P. M., Ardila-Robayo, M. C. (1997). Patrones biogeográficos de las ranas y sapos de Colombia. Revista de la Academia Colombiana de Ciencias Exactas, Físicas y Naturales. 21 (80): 237-248.

Méndez-Narváez, J., Bolívar-G, W. (2016). Complementary ecological approaches to understand decreases in frog diversity in altered Andean ecosystems. South American Journal of Herpetology. 11 (1): 1-11.

Meza-Joya, F. L., Torres, M. (2016). Spatial diversity patterns of Pristimantis frogs in the Tropical Andes. Ecology and Evolution. 6 (7): 1901-1913.

Montes, A. H., Olaya-M, L. A., Castro-H, F. (2004). Incidencia de la perturbación antrópica en la diversidad, la riqueza y la distribución de Eleutherodactylus (Anura: Leptodactylidae) en un bosque nublado del suroccidente Colombiano. Caldasia. 26 (1): 265-274.

Myers, N., Mittermeier, R. A., Mittermeier, C. G., Da Fonseca, G. A., Kent, J. (2000). Biodiversity hotspots for conservation priorities. Nature. 403 (6772): 853-858.

Pough, F. H., Andrews, R. M., Crump, M. L., Savitzky, A. H., Wells, K. D., Brandley, M. C. (2016). Herpetology. 4th edition. Massachusetts, USA: Sinauer Associates, Inc. 591 pp.

Rada, M., Ospina-Sarria, J. J., Guayasamin, J. M. (2017). A taxonomic review of Tan-Brown Glassfrogs (Anura: Centrolenidae), with the description of a new species from southwestern Colombia. South American Journal of Herpetology. 12 (2): 136-156.
Rangel-Churio, O. J. (2006). La biodiversidad de Colombia. Ibagué, Colombia: Universidad del Tolima, 292 pp.

R Core Team. (2016). R: A language and environment for statistical computing, R Foundation for Statistical Computing, Vienna, Austria. Disponible en: http://www.R-project.org/, accedida entre enero y junio de 2018.

Rios-López, N., Aide, M. (2007): Herpetofaunal dynamics during secondary succession. Herpetologica. 63: 35-50.

Rivera-Correa, M., Faivovich. J. (2013). A new species of Hyloscirtus (Anura: Hylidae) from Colombia, with a rediagnosis of Hyloscirtus larinopygion (Duellman, 1973). Herpetologica. 69: 298-313.

Rosenzweig, M. L. (1995). Species diversity in space and time. Cambridge, United Kingdom: Cambridge University Press, $436 \mathrm{pp}$.

Rueda-Almonacid, J. V., Rada, M., Pacheco, Á. A. V. Á., Quevedo, A. (2006). Two new and exceptional poison dart frogs of the genus Dendrobates (Anura: Dendrobatidae) from the Northeastern Flank of the cordillera Central of Colombia. Zootaxa. 1259: 39-54.

Rueda-Almonacid, J.V. (1999). Anfibios y reptiles amenazados de extinción en Colombia. Revista de la Academia Colombiana de Ciencias Exactas, Físicas y Naturales. 23 (Suplemento Especial): 475-497

Rueda-Almonacid, J.V. (2000). La herpetofauna de los "Bosques de Florencia", Caldas: una aproximación a su composición, diversidad y relaciones ecológicas. Corporación Autónoma Regional de Caldas. Bogotá, Colombia: CORPOCALDAS.

Rueda-Almonacid, J. V., Rodríguez-Mahecha, J. V., La Marca, E., Lötters, E., Kahn, T., Angulo, A. (2005). Ranas arlequines. Bogotá, Colombia: Panamericana Formas e impresos S. A. 200 pp.

Ruiz, A. Rueda-Almonacid, J. V. (2008). Batrachochytrium dendrobatidis and chytridiomycosis in anuran amphibians of Colombia. EcoHealth. 5 (1): 27-33.

Ruiz-Carranza, P. M., Lynch, J. D. (1997). Ranas Centrolenidae de Colombia X. Los centrolénidos de un perfil del flanco oriental de la cordillera Central en el Departamento de Caldas. Revista de la Academia Colombiana de Ciencias Exactas, Físicas y Naturales. 21 (81): 541-553.

Russildi, G., Arroyo-Rodríguez, V., Hernández-Ordóñez, O., Pineda, E., Reynoso, V. H. (2016). Species-and communitylevel responses to habitat spatial changes in fragmented rainforests: assessing compensatory dynamics in amphibians and reptiles. Biodiversity and Conservation. 25 (2): 375-392.

Urbina-Cardona, J. N. (2016). Gradientes andinos en la diversidad y patrones de endemismo en anfibios y reptiles de Colombia: Posibles respuestas al cambio climático. Revista Facultad de Ciencias Básicas. 7 (1): 74-91.

Vallan, D. (2002). Effects of anthropogenic environmental changes on amphibian diversity in the rain forests of eastern Madagascar. Journal of Tropical Ecology. 18 (5): 725-742.

Zermeño-Hernández, I., Pingarroni, A., Martínez-Ramos, M. (2016). Agricultural land-use diversity and forest regeneration potential in human-modified tropical landscapes. Agriculture, Ecosystems \& Environment. 230: 210-220. 